\title{
Euchromatin histone methyltransferase II (EHMT2) regulates the expression of ras-related GTP binding C (RRAGC) protein
}

\author{
Supyong Hwang ${ }^{1}$, Soyoung Kim ${ }^{1}$, Kyungkon Kim ${ }^{1,2,3}$, Jeonghun Yeom ${ }^{2}$, Sojung Park ${ }^{1}$ E Inki Kim ${ }^{1,2,3, *}$ \\ ${ }^{1}$ Biomedical Research Center, ASAN Institute for Life Sciences, ASAN Medical Center, Seoul 05505, ${ }^{2}$ Convergence Medicine Research \\ Center (CREDIT), ASAN Institute for Life Sciences, ASAN Medical Center, Seoul 05505, ${ }^{3}$ Department of Convergence Medicine, \\ University of Ulsan College of Medicine, Seoul 05505, Korea
}

Dimethylation of the histone $\mathrm{H} 3$ protein at lysine residue 9 (H3K9) is mediated by euchromatin histone methyltransferase II (EHMT2) and results in transcriptional repression of target genes. Recently, chemical inhibition of EHMT2 was shown to induce various physiological outcomes, including endoplasmic reticulum stress-associated genes transcription in cancer cells. To identify genes that are transcriptionally repressed by EHMT2 during apoptosis, and cell stress responses, we screened genes that are upregulated by BIX-01294, a chemical inhibitor of EHMT2. RNA sequencing analyses revealed 77 genes that were upregulated by BIX-01294 in all four hepatic cell carcinoma (HCC) cell lines. These included genes that have been implicated in apoptosis, the unfolded protein response (UPR), and others. Among these genes, the one encoding the stress-response protein Ras-related GTPase C (RRAGC) was upregulated in all BIX-01294-treated HCC cell lines. We confirmed the regulatory roles of EHMT2 in RRAGC expression in HCC cell lines using proteomic analyses, chromatin immune precipitation (ChIP) assay, and small guide RNA-mediated loss-of-function experiments. Upregulation of RRAGC was limited by the reactive oxygen species (ROS) scavenger $\mathrm{N}$-acetyl cysteine (NAC), suggesting that ROS are involved in EHMT2-mediated transcriptional regulation of stress-response genes in HCC cells. Finally, combined treatment of cells with BIX-01294 and 5Aza-cytidine induced greater upregulation of RRAGC protein expression. These findings suggest that EHMT2 suppresses expression of the RRAGC gene in a ROS-dependent manner and imply that EHMT2 is a key regulator of stress-responsive gene expression in liver cancer cells. [BMB Reports 2020; 53(11): 576-581]

${ }^{*}$ Corresponding author. Tel: +82-2-3010-2515; Fax: +82-2-3010-2515; E-mail: ik.kim@amc.seoul.kr

https://doi.org/10.5483/BMBRep.2020.53.11.055

Received 16 March 2020, Revised 6 April 2020, Accepted 16 June 2020

Keywords: BIX-01294, EHMT2, Epigenetics, ER stress, RRAGC

\section{INTRODUCTION}

Epigenetic modifications are gene regulatory mechanisms that are independent of changes in DNA sequences (1). This mode of gene regulation can be achieved by means of histone and DNA modifications, such as methylation and acetylation (1). Among these, methylation at lysine residues $4(\mathrm{H} 3 \mathrm{~K} 4)$ and 36 ( $\mathrm{H} 3 \mathrm{~K} 36)$ of histone $\mathrm{H} 3$ are hallmarks of transcriptional activation, whereas methylation of histone $\mathrm{H} 3$ residues at lysines 9 (H3K9) and 27 (H3K27) leads to repression (2). Methylation of histones is accomplished by histone methyltransferases (HMTs) that carry the SET domain, which is responsible for recognition of substrates and transfer of methyl groups to $\varepsilon$-amino groups of specific lysine tails on histone proteins (3).

Euchromatin histone methyl-transferase 2 (EHMT2) is responsible for methylation of histone lysine (K) 9 residues and thereby suppresses various genes (4). Recently, abnormal upregulation of EHMT2 reportedly increased H3K9 methylation in a wide range of cancers, including hepatic cell carcinomas (HCC) (5-8). These reports concluded that EHMT2-mediated silencing of various genes promotes the progression of HCC, suggesting the potential of EHMT2 as a target for future liver-cancer therapies. However, the genes that are regulated by EHMT2mediated silencing mechanisms have not been clearly described in HCC cells. Considering the roles that liver cells play in detoxification, metabolism, and protein secretion, it has been suggested that stress- response-associated genes are regulated by epigenetic mechanisms, such as those involving EHMT2.

In this study, we screened differently expressed genes after treating HCC cells with the small-molecule inhibitor of EHMT2, BIX-01294, and isolated the Ras-related GTPase C (RRAGC) regulator protein of mTOR as a target of EHMT2. Our study suggests that EHMT2 is involved in the regulation of stressresponse genes and mammalian target of rapamycin (mTOR) signaling in HCC cells.

\section{RESULTS}

RNA-sequencing analysis of genes that are upregulated by EHMT2 inhibition

To identify genes that are regulated by EHMT2, we incubated

ISSN: 1976-670X (electronic edition)

Copyright (C) 2020 by the The Korean Society for Biochemistry and Molecular Biology

(c) This is an open-access article distributed under the terms of the Creative Commons Attribution Non-Commercial License (http://creativecommons.org/licenses/by-nc/4.0) which permits unrestricted non-commercial use, distribution, and reproduction in any medium, provided the original work is properly cited. 
four HCC cell lines with the EHMT2 inhibitor BIX-01294 and did RNA-sequencing analysis. We prepared total RNA samples from non-treated and BIX-01294-treated cells and analyzed differences in expression levels. The Venn diagram in Fig. 1A shows the numbers of genes that were commonly and uncommonly upregulated in the four HCC cell lines after 15-h incubation with $10 \mu \mathrm{M}$ BIX-01294. A total of 77 genes were upregulated in all four cell lines. We confirmed the demethylation efficacy of BIX-01294 in all cell lines by comparing the dimethylation level of H3K9 (Supplementary Fig. S1A).

From genes that were upregulated in all cell types, we selected those associated with apoptosis and ER stress. As shown in Fig. 1B, a total of 33 genes included autophagy genes, ER stress-related genes, and apoptosis-related genes. Among the encoded proteins, we identified protein phosphatase 1 regulatory subunit 15A (PPP1R15A), DNA Damage Inducible Transcript 3 (DDIT3), Tribbles Homolog 3 (TRIB3), and Rasrelated GTPase C (RRAGC). Fold changes in gene expression levels are listed in Fig. 1C, and commonly upregulated genes are grouped by their cellular functions in Supplementary Table 2.

\section{BIX-01294 upregulates RRAGC}

To confirm expression levels of the four ER stress- and apop- tosis-related genes identified above, we did quantitative PCR (qPCR) analyses (Fig. 2). Expression levels of all four genes were increased by BIX-01294 in a dose-dependent manner. Moreover, in separate cell culture experiments, the effects of BIX-01294 were more concentration-dependent in SNU475 cells than in HepG2 cells. In the latter cell line, increases in expression levels were negligible with $5-\mu \mathrm{M}$ BIX-01294, but were significant when cells were treated with $10 \mu \mathrm{M}$ BIX01294. Our results are consistent with our RNA-sequencing analysis (Fig. 1) and show that EHMT2 regulates expression levels of stress-response genes, including those involved in apoptosis and ER stress.

\section{BIX-01294 increases RRAGC protein expression}

To find out whether the transcriptional effects of BIX-01294 shown in RNA-sequencing analyses were accompanied by changes in protein expression, we did proteome analyses in BIX-01294-treated HCC cell lines. In these experiments, we incubated SNU475 cells with BIX-01294 as described for RNA-sequencing analyses $(10 \mu \mathrm{M}, 15 \mathrm{~h})$ and prepared cell extracts for analyses of protein expression levels. As shown in Fig. 3A, RRAGC protein expression was significantly increased after BIX-01294 treatments. We were, however, unable to demonstrate similar BIX-01294-mediated increases in protein expres-

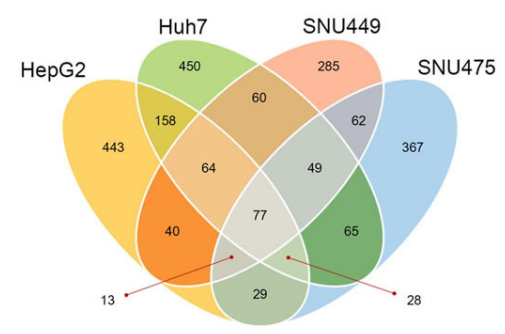

C
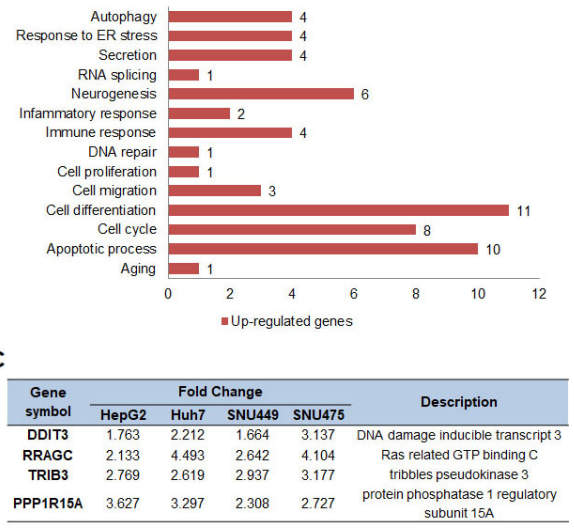

Fig. 1. BIX-01294 upregulates apoptosisand stress-response-related genes; (A) the Venn diagram shows numbers of upregulated genes in BIX-01294-treated HCC cell lines. (B) Genes that were upregulated in all four HCC cells were classified using gene ontology (GO) terms. (C) List of selected genes from (A) and (B) showing induced expression of more than 1.5-fold compared with the non-treated control. We classified 33 genes into each group including duplication.

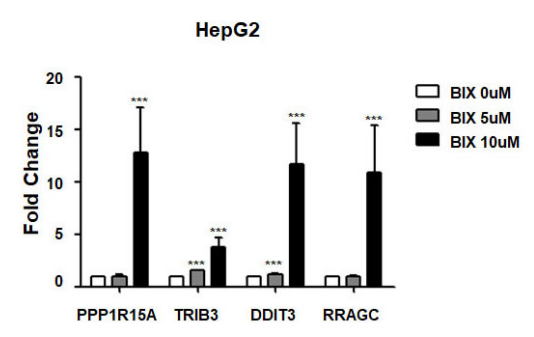

http://bmbreports.org
SNU475

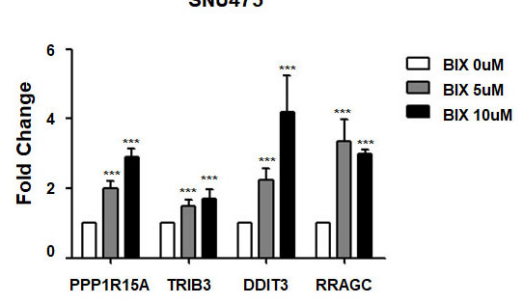

Fig. 2. RRAGC mRNA expression is upregulated by BIX-01294. We incubated two HCC cell lines (HepG2 (A) and SNU-475 (B)) with BIX-01294 at 5 and $10 \mu \mathrm{M}$ for $15 \mathrm{~h}$. We analyzed RNA samples using quantitative reverse transcriptase-polymerase chain reaction (qRT-PCR) analysis. Data are presented as means \pm standard deviations (SD; $n=2$; ***P $<0.001)$. 
A
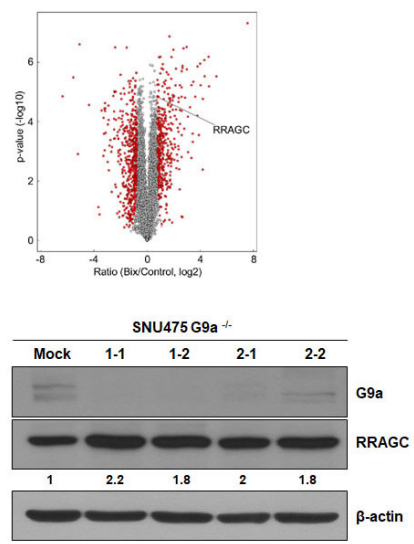

B

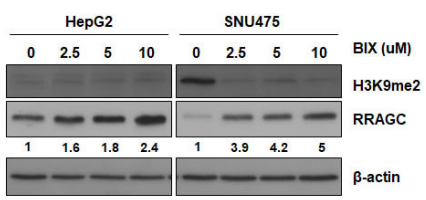

D

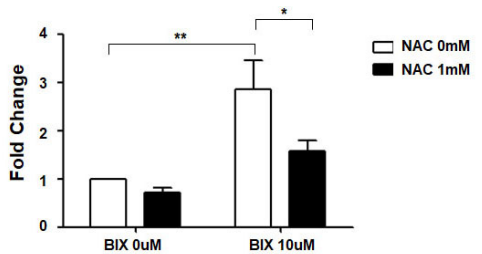

Fig. 3. RRAGC protein expression was induced by BIX-01294 via a ROS-dependent mechanism. We treated HepG2 and SNU475 cells with BIX-01294 at $10 \mu \mathrm{M}$ for $15 \mathrm{~h}$. Elevated RRAGC protein expression levels are indicated in the volcano plot from proteome analyses (A) and in Western blots (B). EHMT2 genes were deleted in SNU475 cells as described in the Materials and Methods, and RRAGC expression was analyzed by Western blotting. Representative Western blotting images are presented from two independent experiments (C). (D) SNU475 cells were pre-treated with N-acetyl cysteine (NAC) for $1 \mathrm{~h}$, and then with BIX-01294 at $10 \mu \mathrm{M}$ for $15 \mathrm{~h}$. We analyzed RRAGC mRNA expression levels using qRT-PCR. Data are presented as means $\pm \mathrm{SD} ; n=2 ; * \mathrm{P}<0.05, * * \mathrm{P}<0.01$.

sion levels of the other three.

In Western blotting analyses, we confirmed that RRAGC expression levels are increased after EHMT2 inhibition. As shown in Fig. 3B, BIX-01294 treatments increased RRAGC protein expression levels in a dose-dependent manner (Fig. 3B) in HepG2 and SNU475 cells. Thus, we examined the effects of EHMT2 on RRAGC protein expression in EHMT2 knockout HCC cells that were produced using Crispr/Cas9 technology. In engineered SNU475 cells that lacked the EHMT2 gene, RRAGC protein expression was increased, as indicated by the Western blots in Fig. 3C. Moreover, all four puromycin-resistant clones had increased expression levels of the RRAGC protein. These results suggest that RRAGC mRNA and consequent protein expression are regulatory targets of EHMT2.

Previous reports showed that reactive oxygen species (ROS) are involved in EHMT2-mediated gene expression (9). Thus, to find out the roles of ROS in RRAGC regulation by EHMT2, we incubated HCC cells with BIX-01294 with or without the ROS scavenger $\mathrm{N}$-acetyl cysteine (NAC). In subsequent qRT-PCR analysis of BIX-01294-treated SNU-475 cells, NAC significantly reduced the upregulation of RRAGC gene expression (Fig. 3D). Taken together, our data indicate that EHMT2 inhibition leads to upregulation of RRAGC mRNA and protein in a ROS-dependent manner.

\section{EHMT2 cooperates with DNA methyltransferase 1 (DNMT1)} to suppress transcriptional activation of RRAGC

It was recently reported that EHMT2 can directly bind DNMT1 with resulting formation of a protein complex to achieve synergistic suppression of gene transcription (10), suggesting that hypermethylation of $\mathrm{H} 3 \mathrm{~K} 9$ and DNA can be linked to the suppression of a target gene that has a specific function in cell physiology (11). The result presented in a previous report emphasizes this possibility (12).

We wondered whether EHMT2 might work with DNMT1 to silence gene expression of RRAGC. We incubated SNU475 cells with 5-Aza-cytidine, a small-molecule inhibitor of DNMT1 with or without BIX-01294 and examined RRAGC protein expression using Western blotting. As shown in Fig. 4A, 5-Aza-cytidine could restore expression of RRAGC. Moreover, combined incubation of cells with 5-Aza-cytidine and BIX01294 induced greater expression of RRAGC than from the incubation of either BIX-01294 or 5-Aza-cytidine alone. Moreover, in EHMT2 knockout cells, 5-Aza-cytidine could restore expression of RRAGC, suggesting that RRAGC is a common target of EHMT2 and DNMT1 (Supplementary Fig. S1B).

\section{Chemical inhibition of EHMT2 induces transcriptional activation of RRAGC}

To better characterize the epigenetic expression regulation of RRAGC by EHMT2, we employed a ChIP assay for RRAGC promoter. We found that the interactions of H3K9me2 and EHMT2 with the RRAGC promoter were decreased, whereas RNA polymerase II binding was increased by BIX-01294 treatment (Fig. 4B). This result suggested that methylation of H3K9 by EHMT2 can downregulate the expression of RRAGC in HCC cells.

\section{DISCUSSION}

Cells respond to extracellular stress by means of crosstalk between cell-signaling pathways, and changes in gene expression 
A

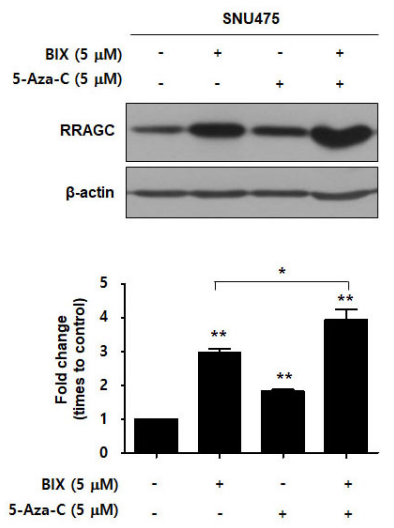

B

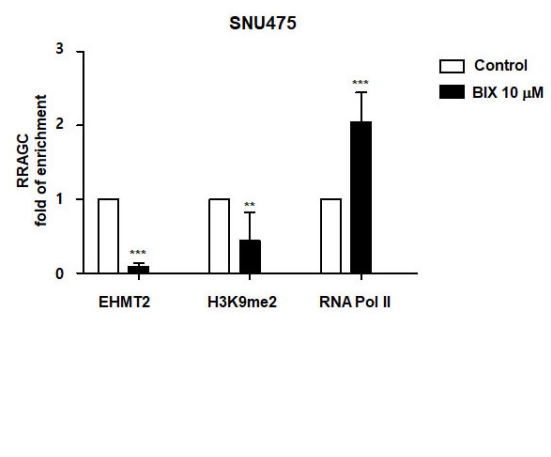

Fig. 4. (A) Coordination of EHMT2 and DNMT1 for the regulation of RRAGC expression. Upper panel : western blot image for the detection of RRAGC expression by treatment of SNU475 cells with BIX-01294 $(5 \mu \mathrm{M})$, 5-Aza-cytidine $(5 \mu \mathrm{M})$, or a combination of both. A representative Western blotting image is presented from three independent experiments. Lower panel: densitometric analysis of protein bands are shown. Data are presented as means $\pm \mathrm{SE} ; n=2(* \mathrm{P}<0.05, * * \mathrm{P}<0.01)$. (B) Transcriptional activation of RRAGC by BIX-01294. SNU475 cells were incubated with $10 \mu \mathrm{M}$ BIX-01294 for $4 \mathrm{~h}$. Treated cells were subjected to ChIP analysis done with anti-EHMT2 antibody, anti-H3K9me2 antibody, and anti-RNA polymerase II antibodies. The real-time quantification of the RRAGC promoter sequences was presented as fold enrichment. Data are presented as means $\pm \mathrm{SD} ; n=2\left({ }^{* * P}<0.01,{ }^{* * * P}<0.001\right)$.

are core to cell stress responses. Because misregulation of gene expression can result in abnormal cell fates, expression levels are tightly regulated by means of transcriptional, translational, and epigenetic regulatory mechanisms. Among these modes of regulation, epigenetic regulatory mechanisms are the most recently identified. Lysine residues of histone proteins are the main sites of epigenetic gene regulation and act as substrates for epigenetic enzymes, including acetyl transferase, deacetylase, methyl transferase, and demethylase (2).

In this report, we screened genes that are upregulated following treatments with the small-molecule inhibitor of the histone methyltransferase EHMT2, which methylates lysine residue 9 of histone $\mathrm{H} 3$ (H3K9) (13). Previous studies show that EHMT2 regulates cell stress responses, including autophagy (14), apoptosis $(14,15)$, and the UPR (9). In agreement, our RNA-sequencing analyses identified genes that are upregulated by EHMT2 inhibition and are involved in the aforementioned cellular responses. A total of 77 genes were upregulated by BIX-01294 in all tested HCC cell lines (Fig. 1). After careful review of the functions of these commonly upregulated genes, we selected four with known roles in apoptosis and ER stress (or the UPR). In quantitative real-time PCR assays, these genes were upregulated by BIX-01294 treatments (Fig. 2), yet only RRAGC was upregulated the protein level in proteome analyses (Fig. 3A). Because the other candidate proteins (PPP1R15A, TRIB3, and DDIT3) were not detected in proteome analyses, we used Western blotting analyses. In these experiments, PPP1R15A and TRIB3 protein expression levels were not induced by BIX-01294 despite changes in gene expression levels (data not shown). DDIT3 expression was induced by BIX-01294, but not until a later time, when UPR activation was also observed (data not shown in this report).

We also investigated the possible collaboration of EHMT2 with DNMT1 by combined incubation of cells with both BIX-01294 and 5-Aza-cytidine (Fig. 4A and Supplementary Fig. S1B). Our data suggested that EHMT2 can work together with DNMT1 to suppress the expression of RRAGC by a previously identified molecular mechanism $(10,12)$. Moreover, we analyzed the binding of $\mathrm{H} 3 \mathrm{~K} 9 \mathrm{me} 2$ and EHMT2 to the RRAGC promoter by a ChIP assay suggesting the transcriptional inactivation of RRAGC by EHMT2 (Fig. 4B).

RRAGC is considered to be a regulator of mTOR signaling (16-18) and activated mTOR pathway. Because RRAGC-activated mTORC1 reportedly inhibited autophagosome formation caused by accumulation of p62 (19), we investigated the relationship between RRAGC and BIX-01294-induced cell death in loss-of-function studies using siRNAs. We observed no effect of RRAGC knockdown on the induction of cell death in BIX-01294-treated HCC cells (Supplementary Fig. S2A and S2C), but observed simultaneous accumulation of LC3B and p62, indicating that cyto-protective autophagy was disrupted (Supplementary Fig. 2B). These results are consistent with previous studies showing that BIX-01294 induces autophagy but results in apoptosis (14) and that RRAGC-induced activation of mTORC1 leads to inhibition of autophagosome formation (19).

Collectively, our data fail to delineate the roles of RRAGC following upregulation by EHMT2 inhibition, but they suggest that mTOR activity is regulated by Ras-related GTP binding proteins, such as RRAGC. Further studies of the roles of RRAGC will elucidate the mechanisms of EHMT2 in cells 
exposed to extracellular stress.

\section{MATERIALS AND METHODS}

\section{Reagents and cell culture}

We purchased BIX-01294 from Sigma Aldrich (St. Louis, $\mathrm{MO}$ )and four HCC cell lines from American Type Cell Culture (Manassas, VA). All cells were maintained at $37^{\circ} \mathrm{C}$ in humidified incubators containing $5 \% \mathrm{CO}_{2}$. We cultured SNU449 and SNU475 cells in RPMI-1640 media, whereas Huh7 and HepG2 cells were maintained in Dulbecco's modified Eagle medium. All culture media were supplemented with $10 \%$ fetal bovine serum, $2 \mathrm{mM}$ L-glutamine, and 100-unit/ml antibiotics.

\section{RNA-sequencing analysis}

We did RNA isolation, library preparation and RNA sequencing, and data analyses as described in a previous publication (20). We collected data for downregulated genes from a previous study of three cell lines (20) and generated the dataset for upregulated genes from the four present cell lines.

\section{Western blotting}

We prepared whole-cell extracts in extraction buffer supplemented with phosphatase and protease cocktail inhibitors. After bicinchoninic acid assays (Pierce, Rockford, IL, USA), $25-\mu \mathrm{g}$ cell extracts in $1 \mathrm{X}$ Lamaeli sample buffer were separated using sodium dodecyl sulfate polyacrylamide gel electrophoresis (SDS-PAGE) and were transferred to polyvinyl difluoride membranes (Millipore, Billerica, MA). Next, we blocked the membranes using $5 \%$ blocking reagent diluted in Trisbuffered saline with $0.1 \%$ TWEEN 20 (TBS-T). Membranes were then incubated with primary antibody (dilution 1:5000-1: $1,000)$ for $2 \mathrm{~h}$ at room temperature and were washed with TBS-T three times. After incubation with secondary antibody $(1: 5,000)$ and washing with TBS-T, target protein-antibody complexes were visualized using horse radish peroxidase reactions and an enhanced chemiluminescence detection kit (DyneBio, Seoul, Korea).

\section{Primary antibodies for Western blotting}

Anti-H3K9me2 antibody (\#4658), anti-EHMT2 antibody (\#3306), anti-RRAGC antibody (\#5466) were purchased from Cell Signaling Technology (Danvers, MA). We used Anti- $\beta$-actin antibody (NB600-501, Novus, Centennial, CO) as a loading control.

\section{Chromatin Immunoprecipitation (ChIP) assay}

We did a ChIP assay in SNU475 control cells and BIX01294-treated cells using an EZ-Magna ChIP ${ }^{\mathrm{TM}}$ AVG Chromatin Immunoprecipitation Kit (Millipore, Temecula, CA) according to the manufacturer's instructions. EHMT2 antibody (3306, Cell signaling, Danvers, MA), H3K9me2 antibody (17-648, Millipore, Temecula, CA), and RNA Pol II antibody (05-623B, Millipore, Temecula, CA) were used. To detect EHMT2,
H3K9me2 and RNA Pol II enrichment in RRAGC promoter, we did quantitative real-time PCR. The primer sequence used for qPCR was RRAGC Forward 5'- CCCATACCTTTGCACATCCC$3^{\prime}$ and RRAGC Reverse 5'- TGCTCGGGAGTTGAATGAGA-3'.

\section{Quantitative real-time PCR}

We isolated total RNA from cultured cells using Trizol Reagent and then prepared cDNAs using RivertAid First Strand cDNA Synthesis Kits (K1622, Thermo Scientific ${ }^{\text {TM }}$, Waltham, MA). We did quantitative real-time PCR using a LightCycler $^{\circledR} 480$ Instrument II (Roche Applied Science, Penzberg, Germany) with LightCycler ${ }^{\circledR} 480$ SYBR Green I Master mix (Roche Diagnostics, Mannheim, Germany). Primer sequences are listed in the Supplementary Table 1. Glyceraldehyde phosphate dehydrogenase (GAPDH) was used as an endogenous normalization control. Data were analyzed using the comparative method $(\Delta \Delta \mathrm{Ct})$.

\section{Construction of EHMT2 knockout cell lines}

We constructed EHMT2 knockout cell lines using small guided RNA (20). The sequences for sgRNAs that were used to delete EHMT2 were as follows: EHMT2 sgRNA\#1 - EHMT2-RG1F 5'-caccAGAAGTGACCCTGACGAAAG-3' and EHMT2-RG1R 5'-aaacCTTTCGTCAGGGTCACTTCT-3' and EHMT2 sgRNA\#2 EHMT2 RG2F 5'-CaCCCTACTATGATTCCTACTCTG-3' and EHMT2 RG2R 5'-aaacCAGAGTAGGAATCATAGTAG-3'. After producing lentivirus vectors, SNU475 cells were infected, and transfected clones were selected by incubating cells with puromycin $(2 \mu \mathrm{g} / \mathrm{ml})$. We monitored the efficiency of EHMT2 gene knockout using Western blotting.

\section{Proteomics data: sample preparation for proteome identification/quantification}

Each SNU475 cell pellet was lysed in $400 \mu \mathrm{L}$ of lysis buffer containing 5\% SDS and 50-mM triethylammonium bicarbonate $(\mathrm{pH}$ 7.55) using a sonicator. Lysates were cleared by centrifugation and we measured protein concentrations using a $\mathrm{BCA}$ assay. The equal amounts of proteins $(300 \mu \mathrm{g})$ were subjected to $\mathrm{S}$ Trap-based tryptic digestion with a modified trypsin/lysC mixture (Promega, Madison, WI).

\section{Proteomic data: nano-liquid chromatography-electrospray and ionization-tandem mass-spectral (LC-ESI-MS/MS) analysis \\ We analyzed peptide mixtures on a Dionex UltiMate 3000 RSLC nano LC system (Thermo Scientific, Waltham, MA) coupled to an Q Exactive Plus mass spectrometer (Thermo Scientific, Waltham, MA). Tryptic peptides were reconstituted using $0.1 \%$ formic acid and were loaded. We then separated peptides on an Acclaim PepMap RSLC analytical column (NanoViper C18, Thermo Scientific) over 200 min using a $0 \%-40 \%$ acetonitrile gradient. Next, we acquired mass spectra in a data-dependent mode (m/z 350-1800) and with 20 data-dependent MS/MS scans. All biological samples were}


analyzed in three technical replicates.

Proteomic data: database search and label-free quantification We examined the acquired MS/MS spectra using the SequestHT analysis tool on proteomics discoverer (Thermo Fisher, Waltham, MA) in the SwissProt. Subsequently, we did label-free quantification using peak intensity analysis for unique and razor peptides of protein. Data were normalized to total peptide quantities.

\section{Statistical analysis of proteome data}

To analyze relative abundances of proteins between samples, we used Perseus software. Normalized protein abundances were $\log 2$ transformed, and technical replicates were grouped, with a minimum of three valid values. We identified differences between samples using $t$-tests with a permutation-based false discovery by cut-off rate of 0.05 .

\section{Statistical analysis}

All results are presented as means \pm standard deviations (SD) or standard error (SE), and significance differences were confirmed using $t$-test.

\section{ACKNOWLEDGEMENTS}

This study was supported by a grant from the Bio and Medical Technology Development Program of the National Research Foundation (2017M3A9G7072719) and by the intramural research program of ASAN Institute for Life Sciences (2017721). We thank the core facilities of HTS and CPC laboratory in the ConveRgence mEDIcine research center (CREDIT), and Asan Medical Center for the use of their shared equipment, services, and expertise.

\section{CONFLICTS OF INTEREST}

The authors have no conflicting interests.

\section{REFERENCES}

1. Chen Z, Li S, Subramaniam S, Shyy JY and Chien S (2017) Epigenetic Regulation: A new frontier for biomedical engineers. Annu Rev Biomed Eng 19, 195-219

2. Hyun K, Jeon J, Park K and Kim J (2017) Writing, erasing and reading histone lysine methylations. Exp Mol Med 49, e324

3. Herz HM, Garruss A and Shilatifard A (2013) SET for life: biochemical activities and biological functions of SET domaincontaining proteins. Trends Biochem Sci 38, 621-639

4. Fritsch L, Robin P, Mathieu JR et al (2010) A subset of the histone H3 lysine 9 methyltransferases Suv39h1, G9a, GLP, and SETDB1 participate in a multimeric complex. Mol Cell 37, 46-56

5. Casciello F, Windloch K, Gannon F and Lee JS (2015) Functional role of G9a histone methyltransferase in cancer. Front Immunol 6, 487
6. Kondo Y, Shen L, Suzuki S et al (2007) Alterations of DNA methylation and histone modifications contribute to gene silencing in hepatocellular carcinomas. Hepatol Res 37, 974-983

7. Yokoyama M, Chiba T, Zen Y et al (2017) Histone lysine methyltransferase G9a is a novel epigenetic target for the treatment of hepatocellular carcinoma. Oncotarget 8, 21315-21326

8. Wei L, Chiu DK, Tsang FH et al (2017) Histone methyltransferase G9a promotes liver cancer development by epigenetic silencing of tumor suppressor gene RARRES3. J Hepatol 67, 758-769

9. Kim SY, Hong M, Heo SH et al (2018) Inhibition of euchromatin histone-lysine $\mathrm{N}$-methyltransferase 2 sensitizes breast cancer cells to tumor necrosis factor-related apoptosis-inducing ligand through reactive oxygen speciesmediated activating transcription factor 4-C/EBP homologous protein-death receptor 5 pathway activation. Mol Carcinog 57, 1492-1506

10. Esteve PO, Chin HG, Smallwood A et al (2006) Direct interaction between DNMT1 and G9a coordinates DNA and histone methylation during replication. Genes Dev 20, 3089-3103

11. Jones PA and Baylin SB (2002) The fundamental role of epigenetic events in cancer. Nat Rev Genet 3, 415-428

12. Park SE, Yi HJ, Suh N et al (2016) Inhibition of EHMT2/ G9a epigenetically increases the transcription of Beclin-1 via an increase in ROS and activation of NF-kappaB. Oncotarget 7, 39796-39808

13. Stewart MD, Li J and Wong J (2005) Relationship between histone $\mathrm{H} 3$ lysine 9 methylation, transcription repression, and heterochromatin protein 1 recruitment. Mol Cell Biol 25, 2525-2538

14. Kim Y, Kim YS, Kim DE et al (2013) BIX-01294 induces autophagy-associated cell death via EHMT2/G9a dysfunction and intracellular reactive oxygen species production. Autophagy 9, 2126-2139

15. Huang Y, Zou Y, Lin L, Ma X and Huang X (2017) Effect of BIX-01294 on proliferation, apoptosis and histone methylation of acute T lymphoblastic leukemia cells. Leuk Res 62, 34-39

16. Sancak Y, Peterson TR, Shaul YD et al (2008) The Rag GTPases bind raptor and mediate amino acid signaling to mTORC1. Science 320, 1496-1501

17. Kim E, Goraksha-Hicks P, Li L, Neufeld TP and Guan KL (2008) Regulation of TORC1 by Rag GTPases in nutrient response. Nat Cell Biol 10, 935-945

18. Jewell JL, Russell RC and Guan KL (2013) Amino acid signalling upstream of mTOR. Nat Rev Mol Cell Biol 14, 133-139

19. Guha $P$, Kaptan E, Gade $P$, Kalvakolanu DV and Ahmed H (2017) Tunicamycin induced endoplasmic reticulum stress promotes apoptosis of prostate cancer cells by activating mTORC1. Oncotarget 8, 68191-68207

20. Namgung Y, Kim SY and Kim I (2019) Down-regulation of survivin by BIX-01294 pretreatment overcomes resistance of hepatocellular carcinoma cells to TRAIL. Anticancer Res $39,3571-3578$ 